\title{
Photon emission from out of equilibrium dissipative parton plasma
}

\author{
Jitesh R. Bhatt* and V. Sreekanth ${ }^{\dagger}$ \\ Theoretical Physics Division, Physical Research Laboratory, Navrangpura, Ahmedabad, India - 380009
}

(Dated: November 30, 2018)

\begin{abstract}
Using the second order Israel-Stewart hydrodynamics we discuss the effect of viscosity on photon production in a parton plasma created in relativistic heavy ion collisions. We find that photon production rates can enhance by several factors due to the viscous effect in a chemically nonequilibrated plasma.

PACS numbers:
\end{abstract}

A strongly coupled quark-gluon plasma (sGGP) or a matter in a perfect fluid state is widely expected to be produced in recent Relativistic Heavy Ion Collider (RHIC) experiments. Recent measurements has shown that the matter flows very rapidly at the time of its breakup into the freely streaming hadronic matter. Also the measurements of the elliptical flow parameter $v_{2}$ show a strong collectivity in the flow [1, 2, 3]. This would imply that the QGP can have a very low shear viscous stress and the ratio of its shear viscosity $\eta$ to the entropy density $s$ i.e. $\eta / s$ should not be much larger than the lower bound $1 / 4 \pi[4]$. This led to a conjecture that the QGP formed at RHIC is the most perfect-fluid found in nature [5]. There has been a lot of attempts to determine the viscosity of sQGP [5, 6, 7, [8, [9, 10]. The first order theory of viscous hydrodynamics is known to give unphysical results. For example, when the Navier-Stokes equations were applied to the one dimensional boost invariant expanding flow [11], one finds the expression for the temperature to be

$$
T(\tau)=T_{0}\left(\frac{\tau_{0}}{\tau}\right)^{1 / 3}\left[1+\frac{2 \eta}{3 s \tau_{0} T_{0}}\left(1-\left(\frac{\tau_{0}}{\tau}\right)^{2 / 3}\right)\right]
$$

This describes reheating of the flow and $T$ has a maximum at time $\tau_{\max }=\tau_{0}\left(\frac{1}{3}+\frac{s}{\eta} \frac{\tau_{0} T_{0}}{2}\right)^{-3 / 2}$. This is an unphysical behaviour and the first order viscous dynamics is known to have such problems [12, 13]. The second order hydrodynamics approach developed in the spirit of Israel and Stewarts [14] removes such artifacts. The second order viscous hydrodynamics later developed and applied in the context of heavy-ions collisions by several authors $[15,16,17,18,19,20,21,22]$.

It would be interesting to study the role that viscosity can play on the plasma signals. Hard photons are one such promising source that can provide information about the thermodynamical state of the plasma at time of their production. The plasma created in the heavy-ion collisions is expected to be in a state of chemical nonequilibrium. The photon emission from such a plasma has been studied within the framework of ideal hydrodynamics by earlier workers 23, 24, 25, 26]. In this paper we study the photon production using causal hydrodynamics of Israel-Stewart [14].

In the center of the fireball in a nuclear collision the viscous stress-energy tensor in the local comoving frame has the form [16, 27, 28]:

$$
T^{\mu \nu}=\left(\begin{array}{cccc}
\varepsilon & 0 & 0 & 0 \\
0 & P_{\perp} & 0 & 0 \\
0 & 0 & P_{\perp} & 0 \\
0 & 0 & 0 & P_{z}
\end{array}\right)
$$

with the transverse and longitudinal pressure

$$
\begin{aligned}
P_{\perp} & =P+\frac{1}{2} \Phi \\
P_{z} & =P-\Phi
\end{aligned}
$$

Here $P$ denotes the (isotropic) pressure in thermal equilibrium, $\Phi$ denotes the non-equilibrium contributions to the pressure coming from shear stress. We ignore the bulk viscosity in the relativistic limit when the equation of state

\footnotetext{
* email: jeet@prl.res.in

$\dagger$ email: skv@prl.res.in
} 
$p=\epsilon / 3$ is obeyed [29]. However, the bulk viscosity can be important near the critical temperature [30, 31]. The shear tensor in that frame takes the form $\pi^{i j}=\operatorname{diag}(\Phi / 2, \Phi / 2,-\Phi)$ consistent with the symmetries in the transverse directions.

To describe evolution of the energy density and the viscous stress $\phi$ we use second order dissipative hydrodynamics of Israel-Stewart [14, 33, 34, 35]:

$$
\begin{aligned}
\frac{\partial \varepsilon}{\partial \tau} & =-\frac{1}{\tau}(\varepsilon+P-\Phi) \\
\frac{\partial \Phi}{\partial \tau} & =-\frac{\Phi}{\tau_{\pi}}-\frac{1}{2} \Phi\left(\frac{1}{\tau}+\frac{1}{\beta_{2}} T \frac{\partial}{\partial \tau}\left(\frac{\beta_{2}}{T}\right)\right)+\frac{2}{3} \frac{1}{\beta_{2} \tau}
\end{aligned}
$$

where $\beta_{2}=9 /(4 \varepsilon)$ and $\tau_{\pi}=2 \beta_{2} \eta$ denotes the relaxation time. Equations(3-4) are written in the local rest frame using hydrodynamic velocity $u^{\mu}=\frac{1}{\tau}(t, 0,0, z)$, where $\tau=\sqrt{t^{2}-z^{2}}[11]$. Equation of state is required to solve these equations. We use ultra-relativistic equation of state : $P=\frac{1}{3} \epsilon$.

To describe the chemical non-equilibration while maintaining the kinetic equilibrium, one can use the parton distribution [36],

$$
f(k, T)_{q, g}=\lambda_{q, g}(\tau) \frac{1}{e^{\mathbf{u} \cdot \mathbf{k} / T(\tau)} \pm 1}
$$

where, $u^{\mu}$ is the four-velocity of the local comoving reference frame. The temperature $T$ is a time-dependent quantity and the distribution is multiplied by time and another dependent quantity called fugacity $\lambda_{q, g}(\tau)$ to describe deviations from the chemical equilibrium. The fugacity parameter become unity when the chemical-equilibrium is reached and in general it has the range $0 \leq \lambda_{q, g} \leq 1$. The scattering processes $g g \leftrightarrow g g g$ and $g g \leftrightarrow q \bar{q}$ give the most dominant mechanism for the chemical equilibration. The master equations describing evolution the parton density are given by

$$
\begin{aligned}
& \partial_{\mu}\left(n_{g} u^{\mu}\right)=\frac{1}{2} \sigma_{3} n_{g}^{2}\left(1-\frac{n_{g}}{\tilde{n}_{g}}\right)-\frac{1}{2} \sigma_{2} n_{g}^{2}\left(1-\frac{n_{q}^{2} \tilde{n}_{g}^{2}}{\tilde{n}_{q}^{2} n_{g}^{2}}\right), \\
& \partial_{\mu}\left(n_{q} u^{\mu}\right)=\frac{1}{2} \sigma_{2} n_{g}^{2}\left(1-\frac{n_{q}^{2} \tilde{n}_{g}^{2}}{\tilde{n}_{q}^{2} n_{g}^{2}}\right),
\end{aligned}
$$

where $\tilde{n}_{i}(i=q, g)$ is parton density with unit fugacity [37] and $\sigma_{2}=\langle\sigma(g g \leftrightarrow q \bar{q})\rangle$ and $\sigma_{3}\langle\sigma(g g \leftrightarrow g g g)\rangle$ are thermally averaged scattering cross sections. It should be noted here that when equation for $n_{g}$ and $n_{q}$ are added one gets the total number density $n$ and the term with $\frac{1}{2} \sigma_{2} n_{g}^{2}\left(1-\frac{n_{q}^{2} \tilde{n}_{g}^{2}}{\tilde{n}_{q}^{2} n_{g}^{2}}\right)$ will drop out. This is because due to the the scattering process $g g \leftrightarrow q \bar{q}$ loss in the gluon density is equal to the gain in quark density and vice verse.

$\epsilon$ and $n$ can be calculated using equation (5) as given below

$$
n=\left(\lambda_{g} a_{1}+\lambda_{q} b_{1}\right) T^{3}, \quad \epsilon=\left(\lambda_{g} a_{2}+\lambda_{q} b_{2}\right) T^{4}
$$

where $a_{1}=16 \xi(3) / \pi^{2}, a_{2}=8 \pi^{2} / 15$ for the gluons and $b_{1}=9 \xi(3) N_{f} / \pi^{2}, b_{2}=7 \pi^{2} N_{f} / 20$ for the quarks. Using equations (3-4,6-8) following evolution equations for $T, \lambda_{q, g}$ and $\Phi$ can be obtained

$$
\begin{aligned}
\frac{\dot{T}}{T}+\frac{1}{3 \tau} & =-\frac{1}{4} \frac{\dot{\lambda}_{g}+b_{2} / a_{2} \dot{\lambda}_{q}}{\lambda_{g}+b_{2} / a_{2} \lambda_{q}}+\frac{\Phi}{4 \tau} \frac{1}{\left(a_{2} \lambda_{g}+b_{2} \lambda_{q}\right) T^{4}} \\
\dot{\Phi}+\frac{\Phi}{\tau_{\pi}} & =\frac{8}{27 \tau}\left[a_{2} \lambda_{g}+b_{2} \lambda_{q}\right] T^{4}-\frac{\Phi}{2}\left[\frac{1}{\tau}-5 \frac{\dot{T}}{T}-\frac{\dot{\lambda}_{g}+b_{2} / a_{2} \dot{\lambda}_{q}}{\lambda_{g}+b_{2} / a_{2} \lambda_{q}}\right] \\
\frac{\dot{\lambda_{g}}}{\lambda_{g}}+3 \frac{\dot{T}}{T}+\frac{1}{\tau} & =R_{3}\left(1-\lambda_{g}\right)-R_{2}\left(1-\frac{\lambda_{q}^{2}}{\lambda_{g}^{2}}\right) \\
\frac{\dot{\lambda_{q}}}{\lambda_{q}}+3 \frac{\dot{T}}{T}+\frac{1}{\tau} & =R_{2} \frac{a_{1}}{b_{1}}\left(\frac{\lambda_{g}}{\lambda_{q}}-\frac{\lambda_{q}}{\lambda_{g}}\right)
\end{aligned}
$$

where, the rates $R_{2}=0.24 N_{f} \alpha_{s}^{2} \lambda_{g} T \ln \left(5.5 / \lambda_{g}\right)$ and $R_{3}=2.1 \alpha_{s}^{2} T\left(2 \lambda_{g}-\lambda_{g}^{2}\right)^{1 / 2}$ are defined as in Ref. [36, 37]. We would like to note that our gluon fugacity equation (11) differs from that given in Ref. [36, 37] by a fctor of two 
in second term in right hand side. We believe this is a typographical error. In equation (9) the first term on left hand side is due to expansion of the plasma, while on the right hand side the first term describes effect of chemical nonequilibrium and second term is due to the presence of (causal) viscosity. The last term in parenthesis of equation (10) arises because of the chemical nonequilibrium process. It should be noted that equation (9) differ from that considered in Ref. [38]. In their treatment first order viscous hydrodynamics is used which does not require time evolution of $\Phi$. However such treatment give unphysical results like reheating artifact [12] as mentioned before.

Elastic $(g g \leftrightarrow g g)$ as well as nonelastic processes like $g g \leftrightarrow g g g$ can contribute to the shear viscosity. Shear viscosity coefficient was recently calculated for the inelastic process in the presence of chemical nonequilibrium in Ref. [35]. It was shown that $\eta / T^{3} \simeq n_{g} / T^{3} \simeq \lambda_{g}$. From this one can write [34]

$$
\tau_{\pi}=\frac{9}{2 \varepsilon} \lambda_{g} T^{3} .
$$

It ought to be mentioned that this viscosity prescription was not considered considered in Ref. [38]. Kinetic theory without invoking nonequilibrium process gives $\tau_{\pi}=3 / 2 \pi T$.

Real photons are produced from the annihilation of a quark-antiquark pair into a photon and a gluon $(q \bar{q} \rightarrow g \gamma)$ and by absorption of a gluon by a quark emitting a photon $(q g \rightarrow q \gamma)$. Another source of photon production could be the bremsstrahlung but its effect can be ignored in the lowest order of a perturbation theory. In order to compute the photon production rates one needs to know the underlying amplitude $\mathcal{M}$ of the basic process involving the annihilation or Compton scattering process and the parton distribution functions given by [39, 40]

$$
\begin{aligned}
\frac{d N}{d^{4} x d^{3} p}= & \frac{1}{(2 \pi)^{3} 2 E} \int \frac{d^{3} p_{1}}{(2 \pi)^{3} 2 E_{1}} \frac{d^{3} p_{2}}{(2 \pi)^{3} 2 E_{2}} \frac{d^{3} p_{3}}{(2 \pi)^{3} 2 E_{3}} \\
& \times n_{1}\left(E_{1}\right) n_{2}\left(E_{2}\right)\left[1 \pm n_{3}\left(E_{3}\right)\right] \\
& \times \sum_{i}\left\langle|\mathcal{M}|^{2}\right\rangle(2 \pi)^{4} \delta\left(P_{1}+P_{2}-P_{3}-P\right) .
\end{aligned}
$$

Here $P_{1}$ and $P_{2}$ are the 4-momenta of the incoming partons, $P_{3}$ of the outgoing parton, and $P$ of the produced photon. In equilibrium, the distribution functions $n_{i}\left(E_{i}\right)$ are given by the Bose-Einstein distribution, $n_{B}\left(E_{i}\right)=1 /\left[\exp \left(E_{i} / T\right)-1\right]$, for gluons and by the Fermi-Dirac distribution, $n_{F}\left(E_{i}\right)=1 /\left[\exp \left(E_{i} / T\right)+1\right]$, for quarks, respectively. The factor $\left\langle|\mathcal{M}|^{2}\right\rangle$ is the matrix element of the basic process averaged over the initial states and summed over the final states. The $\sum_{i}$ indicates the sum over the initial parton states. The fugacity factors can enter equation (14) when equation (5) is considered

$$
n_{1}(E) n_{2}(E)\left(1 \pm n_{3}(e)\right) \mapsto \lambda_{1} n_{1} \lambda_{2} n_{2}\left(1 \pm \lambda_{3} n_{3}\right)
$$

This is can be rewritten as

$$
\begin{aligned}
\lambda_{1} n_{1} \lambda_{2} n_{2}\left(1 \pm \lambda_{3} n_{3}\right) & =\lambda_{1} \lambda_{2} \lambda_{3} n_{1} n_{2}\left(1 \pm n_{3}\right) \\
& +\lambda_{1} \lambda_{2}\left(1-\lambda_{3}\right) n_{1} n_{2}
\end{aligned}
$$

In carrying out the momentum integration it is useful to introduce a parameter $k_{c}$ to distinguish between soft and hard momenta of the quark [41]. For the hard part of the photon rate following [23] we take $k_{c}^{2}=2 m_{q}^{2}=$ $0.22 g^{2} T^{2}\left(\lambda_{g}+\lambda_{q} / 2\right)$, where, $m_{q}$ is the quark-thermal-mass which can be obtained from zero momentum limit of quark self-energy in the high temperature limit. The first term on the right hand side of equation (15) can lead to the following photon rate [23] using the Boltzmann distribution functions instead of a quantum mechanical ones:

$$
\left(2 E \frac{d n}{d^{3} p d^{4} x}\right)_{1}=\frac{5 \alpha \alpha_{s} \lambda_{q}^{2} \lambda_{g}}{9 \pi^{2}} T^{2} e^{-E / T}\left[\ln \left(\frac{4 E T}{k_{c}^{2}}\right)-1.42\right] .
$$

Here $\alpha$ and $\alpha_{s}$ are the electromagnetic and the strong interaction coupling constants. The second term in equation (15) will give, under the Boltzmann approximation, the following contribution to the photon rate:

$\left(2 E \frac{d n}{d^{3} p d^{4} x}\right)_{2}=$

$$
\frac{10 \alpha \alpha_{s}}{9 \pi^{4}} T^{2} e^{-E / T}\left\{\lambda_{q} \lambda_{g}\left(1-\lambda_{q}\right)\left[1-2 \gamma+2 \ln \left(4 E T / k_{c}^{2}\right)\right]+\lambda_{q} \lambda_{q}\left(1-\lambda_{g}\right)\left[-2-2 \gamma+2 \ln \left(4 E T / k_{c}^{2}\right)\right]\right\},
$$


The total photon production rate $2 E \frac{d n}{d^{3} p d^{4} x}$ can be obtained by adding equations (16-17), is required to be convoluted with the space time evolution of the heavy-ion collision. We define [23]

$$
\begin{aligned}
\left(2 \frac{d n}{d^{2} p_{\perp} d y}\right)_{y, p_{\perp}} & =\int d^{4} x\left(2 E \frac{d n}{d^{3} p d^{4} x}\right) \\
& =Q \int_{\tau_{0}}^{\tau_{1}} d \tau \tau \int_{-y_{n u c}}^{y_{n u c}} d y^{\prime}\left(2 E \frac{d n}{d^{3} p d^{4} x}\right)
\end{aligned}
$$

where the times after the maximum overlap of the nuclei are $\tau_{0}$ and and $\tau_{1}$ and $y_{n u c}$ is the rapidity of the nuclei. $Q$ is the transverse cross-section of the nuclei and $p_{\perp}$ is the photon momentum in direction perpendicular to the collision axis. For a $A u$ nucleus $Q \sim 180 \mathrm{fm}^{2}$. The quantity $\left(2 E \frac{d n}{d^{3} p d^{4} x}\right)$ is Lorentz invariant and it is evaluated in the local rest frame in equation (18). The photon energy in this frame, i.e in the frame comoving with the plasma, can be written as $p_{\perp} \cosh \left(y-y^{\prime}\right)$.
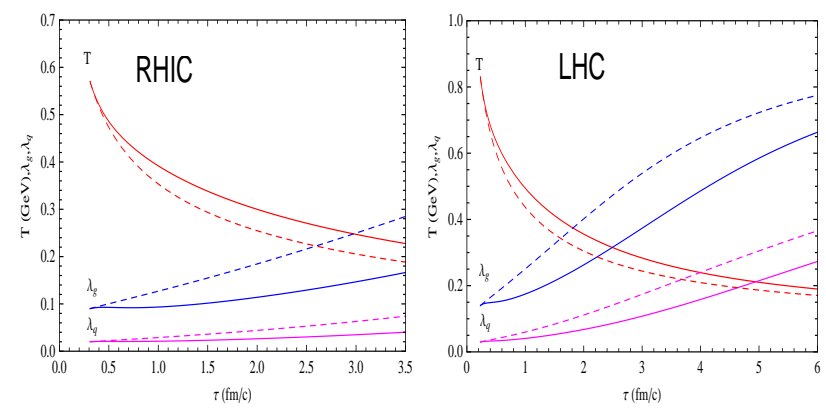

FIG. 1: Temperature, gluon fugacity and quark fugacity for RHIC and LHC. Solid lines indicate the case with the shear viscosity, while the dashed lines correspond to the case without viscosity

In Figure (1), we have shown $T, \lambda_{g}, \lambda_{q}$ as function of time. We have solved the equations (9-12) together with the initial conditions at $\tau_{i s o}$ from HIJING Monte Carlo model [42]. Which are $\lambda_{g}^{0}=0.09, \lambda_{q}^{0}=0.02$ and $T_{o}=0.57 G e V$ for RHIC with $\tau_{i s o}=0.31 \mathrm{fm} / \mathrm{c}$ and $\lambda_{g}^{0}=0.14, \lambda_{q}^{0}=0.03$ and $T_{o}=0.83 \mathrm{GeV}$ for LHC with $\tau_{\text {iso }}=0.23 \mathrm{fm} / \mathrm{c}$. Presence of the causal viscosity decreases the fall of temperature due to expansion and the chemical nonequilibrium. However if one considers the first order theory, there can be unphysical instability. Fugacity of gluons and quarks increase more slowly due to the presence of the viscosity compared to the cases when no viscous effects were included. This is because the chemical equilibration is reached here with falling of the temperature. The temperature can decrease due to the expansion and chemical nonequilibration. The lowering of $T$ can help in attaining chemical equilibrium and which in turn will increase the rate at which the fugacities reach their equilibrium values. Inclusion of the viscosity will slowdown the falling rate of the temperature. Consequently the fugacities will take more time to reach their equilibrium values.
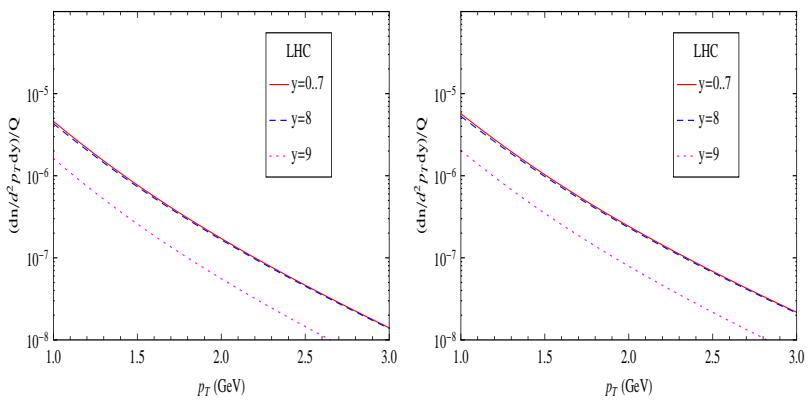

FIG. 2: (Left panel)Photon rate for different rapidities in $L H C$ ( $\left.y_{n u c}=8.8\right)$. (Right panel) Same with the inclusion of viscosity.

We plot photon spectra by using equation (13) for $\tau_{\pi}$ in solving equations (9-12). The figures (2-3) compare the case without viscosity with the case of finite shear viscosity.

Figure (2) shows the photon spectra emitted at fixed rapidities as a function of transverse momenta $p_{\perp}$. The photon flux is normalized with the transverse size of the colliding nuclei $(Q)$. For LHC we take: $\tau_{0}=0.5 f m / c$, 

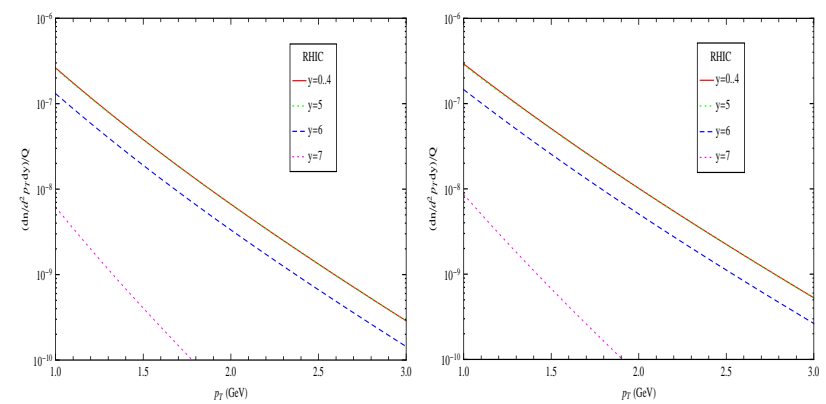

FIG. 3: (Left panel)Photon rate for different rapidities in RHIC ( $\left.y_{n u c}=6.0\right)$. (Right panel) Same with the inclusion of viscosity.
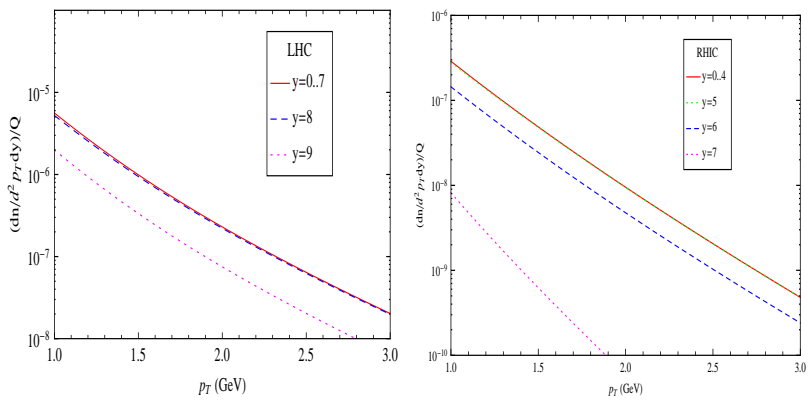

FIG. 4: Photon rate for different rapidities in RHIC $\left(y_{n u c}=6.0\right)$ and LHC $\left(y_{n u c}=8.8\right)$ with kinetic viscosity.

$\tau_{1}=6.25 \mathrm{fm} / \mathrm{c}$ and $y_{n u c}=8.8$. We use equation (13) for $\tau_{\pi}$ in solving equations (9-12). The figure compares the case without viscosity with the case of finite shear viscosity [35].

Figure (3) shows the comparison similar to that of figure (2) but with a set of initial conditions for RHIC: $\tau_{0}=$ $0.7 \mathrm{fm} / \mathrm{c}, \tau_{1}=4 \mathrm{fm} / \mathrm{c}$ and $y_{n u c}=6.0$. For $\alpha_{s}=0.3$, shear viscosity to entropy density ratio $\eta / s \sim 0.29$. Figures (2-3) show that viscous effects enhance the photon flux by a factor (1.5-2).

Finally, we compare the photon fluxes calculated using equation (13) with the fluxes calculated using the kinetic viscosity $\left(\tau_{\pi}=3 / 2 \pi T\right)$. Figure (4) shows the photon flux calculated using the kinetic viscosity prescription for LHC and RHIC. However, we do not find any significant change in the flux for the results obtained using equation (13).

In conclusions, we have studied the second order dissipative hydrodynamics with chemical nonequilibration. We find that the effect of viscosity enhancing the photon flux by a factor ranging between 1.5-2 for the parameter space relevant for LHC and RHIC. Our results are in a broad qualitative agreement with the results obtained in Ref. [38] using the first order theory. We also find that the two viscosity prescriptions with inelastic scattering [35] and the one involving elastic collisions only using kinetic theory give similar results for the photon production rate.

[1] K. H. Ackermann et al. [STAR Collaboration], Phys. Rev. Lett. 86, 402 (2001); C. Adler et al. [STAR Collaboration], ibid. 87 (2001) 182301; 89, 132301 (2002); Phys. Rev. C 66, 034904 (2002); J. Adams et al. [STAR Collaboration], nucl-ex/0409033 J. Adams et al. [STAR Collaboration], Phys. Rev. Lett. 92, 052302 (2004).

[2] K. Adcox et al. [PHENIX Collaboration], Phys. Rev. Lett. 89, 212301 (2002); S. S. Adler et al. [PHENIX Collaboration], ibid. 91, 182301(2003);

[3] B. B. Back et al. [PHOBOS Collaboration], Phys. Rev. Lett. 89, 222301(2002), B. B. Back et al. [PHOBOS Collaboration], Phys. Rev. Lett. 94, 122303 (2005), arXiv:nucl-ex/0406021, B. B. Back et al. Phys. Rev. C 72, 051901 (2005), arXiv:nucl-ex/0407012.

[4] P.K. Kovtun, D.T. Son and A.O. Starinets, Phys. Rev. Lett. 94, 111601 (2005).

[5] T. Hirano and M. Gyulassy, Nucl. Phys. A769, 71 (2006).

[6] G. Baym, H. Monien, C. J. Pethick and D. G. Ravenhall, Phys. Rev. Lett. 64, 1867 (1990).

[7] A. Hasoya and K. Kajantie, Nucl. Phys. B 250, 666 (1985).

[8] H. Heiselberg, Phys. Rev. D 49, 4739 (1994).

[9] P. Arnold, G. D. Moore, L. G. Yaffe, J. High Energy Phys. 11, 001 (2000); J. High Energy Phys. 0305, 051 (2003).

[10] Z. Xu, C. Greiner, Phys. Rev. Lett. 100, 172301 (2008).

[11] J. D. Bjorken, Phys. Rev. D 27, 140 (1983). 
[12] R. Baier, P. Romatschke, and U. A. Wiedemann, Phys. Rev. C 73, 064903 (2006).

[13] W. A. Hiscock and L. Lindblom, Phys. Rev. D 31, 725 (1985).

[14] W. Israel, Annals Phys. 100, 310 (1976); W. Israel and J. M. Stewart, Ann. Phys. 118, 341 (1979).

[15] R. Baier, P. Romatschke, Eur. Phys. J. C 51, 677 (2007).

[16] D. Teaney, Phys. Rev. C 68, 034913 (2003).

[17] U. W. Heinz, H. Song, A. K. Chaudhuri, Phys. Rev. C 73, 034904 (2006).

[18] H. Song, U.W. Heinz, Phys. Rev. C 77, 064901 (2008).

[19] C. E. Aguiar, T. Kodama, T. Osada, Y. Hama, J. Phys. G 27, 75 (2001).

[20] A. Muronga, Phys. Rev. C 76, 014909 (2007).

[21] J. Brachmann, A. Dumitru, J. A. Maruhn, H. Stöcker, W. Greiner, D. H. Rischke, Nucl. Phys. A 619, 391 (1997).

[22] K. Dusling, D. Teaney, Phys. Rev. C 77, 034905 (2008).

[23] C. T. Traxler and M. H. Thoma, Phys. Rev. C 53, 1348 (1996).

[24] D. Dutta, A.K. Mohanty, K. Kumar and R.K. Choudhury, Phys. Rev. C 61, 06491 (2000).

[25] R. Baier, M. Dirks, K. Redlich and D. Schiff, Phys. Rev. D 56, 2548 (1997).

[26] J.L. Long, Z.J. He, Y.G. Ma and B.Liu, Phys. Rev. C 72, 064907 (2005).

[27] A. Muronga, Phys. Rev. Lett. 88, 062302 (2002), [Erratum-ibid. 89, 159901 (2002)].

[28] A. Muronga and D. H. Rischke, (2004) arXiv:nucl-th/0407114.

[29] S. Weinberg, Gravitation and Cosmology, (John Wiley \& Sons, 1972).

[30] R. J. Fries, B. Müller and A. Schäffer, Phys. Rev. C 78, 034913 (2008).

[31] F. Karsch, D. Kharzeev, and K. Tuchin, Phys. Lett. B 663, 217 (2008).

[32] D. Kharzeev and K. Tuchin, arXiv: hep-ph/0705.4280.

[33] U. W. Heinz, (2005), arXiv: nucl-th/0512049.

[34] A. Muronga, Phys. Rev. C 69, 034903 (2004).

[35] A. El, A. Muronga, Z. Xu and C. Greiner, arXiv: hep-ph/0812.27620.

[36] P. Lévai, B. Müller and X. -N. Wang, Phys. Rev. C 51, 3326 (1995).

[37] T. S. Biró, E. van Doorn, B. Müller and X. -N. Wang, Phys. Rev. C 48, 1275 (1993).

[38] A.K. Chaudhuri, J. Phys. G: Nucl. Part. Phys.26 1433 (2000).

[39] T. Peitzmann and M. H. Thoma, Phys. Rept. 364, 175-246 (2002), arXiv: hep-ph/0111114.

[40] C. Gale and K. L. Haglin, arXiv: hep-ph/0306098v3.

[41] J. Kapusta, P. Lichard and D. Seibert, Phys. Rev. D 44, 2774, (1991).

[42] X. N. Wang and M. Gyluassy, Phys. Rev. D 44, 3501, (1991); Comput. Phys. Commun. 83, 307 (1994). 